\title{
Spider silk thread as a fiber optic chemical sensor
}

Luc Thévenaz, Kenny Hey Tow, Desmond Chow, and Fritz Vollrath

Monitoring the properties of light transmitted through a thread of spider silk enables detection of trace amounts of chemical compounds.

Several research teams have recently highlighted how silk can be used as an optical material. Through a regeneration process, it is possible to obtain silk films made from fibers of silk cocoons, by casting a silk fibron solution on an appropriate surface. These transparent films have been used to make optical devices such as diffraction gratings, phase masks, and photonic crystal lattices. ${ }^{1}$ Our research actually involves a more natural approach that uses dragline silk in its pristine condition, extracted directly from the glands of a spider, to serve as a new generation of mechanically strong, biocompatible, and biodegradable optical fibers for new applications in biophotonics. ${ }^{2}$

Silica is commonly used for sensing physical parameters such as temperature, strain, and vibrations, but it is unresponsive to chemical and biological compounds. By contrast, dragline silk fiber is a protein-based biopolymer with functional side groups. Therefore, it is intrinsically sensitive to particular molecules such as proteins and nucleic acids. Moreover, spider silk can operate at the nanoscale level with minimal energy, while occupying a very small footprint for high sensor density, accuracy, and robustness. More specifically, it contains millions of repetitive protein sequences and domains that can interact with a multitude of chemicals via its unique molecular structure, which significantly multiplies the responsivity compared with conventional chemical sensing methods. All these qualities make spider silk an excellent candidate as a photon conveyor and sensing element in a fiber optic chemical sensor system.

To experiment with silk in this way, we used dragline silk from a female Nephila edulis spider, collected by the Oxford Silk Group at the University of Oxford (see Figure 1). By reeling under controlled conditions (reeling speed, humidity, and temperature), we can spin a uniform silk thread with a smooth surface, circular cross section, and homogeneous material properties. ${ }^{3}$

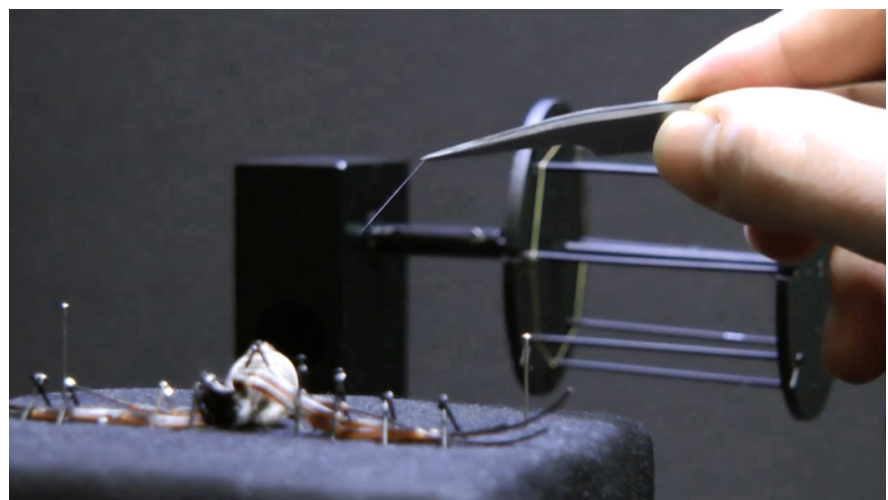

Figure 1. A dragline silk thread being collected from the major ampullate gland of a Golden Orb Weaver spider (Nephila edulis). (Photograph courtesy of Oxford Silk Group.)

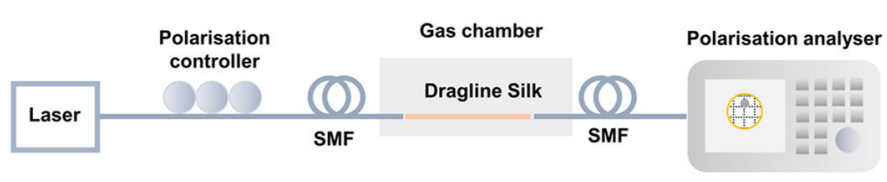

Figure 2. Schematic diagram of the experimental setup used to measure the change in birefringence of the silk fiber material when exposed to different chemical vapors. SMF: Single-mode fiber.

We injected light into the dragline silk, which enabled us to perform a complete optical characterization. We discovered that its transmission window extends from visible light up to $1400 \mathrm{~nm}$, with propagation losses in the order of a few $\mathrm{dB} / \mathrm{cm}$ over the whole spectrum, and more crucially, a high birefringence of $10^{-2}$ to $10^{-3}$.

Silk fibers are held together by reversible hydrogen bonding. These weak bonds can be broken under the influence of polar molecules, thereby causing the molecular chains to disorient and coil-uncoil, and modifying the overall degree of molecular orientation. From an optical perspective, this is translated into a change in the silk fiber's material birefringence, which can be

Continued on next page 


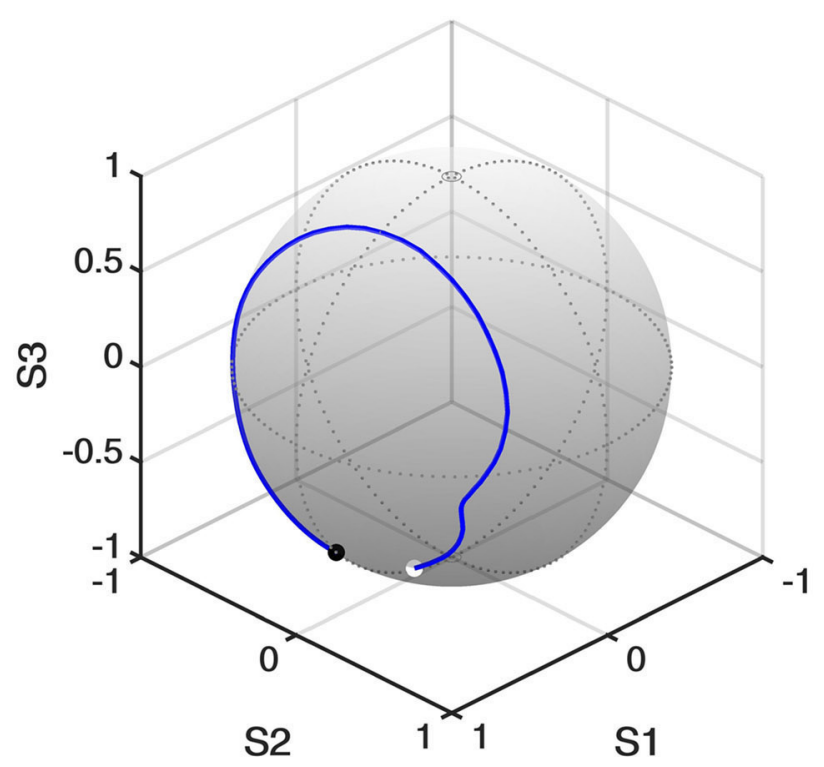

(a)

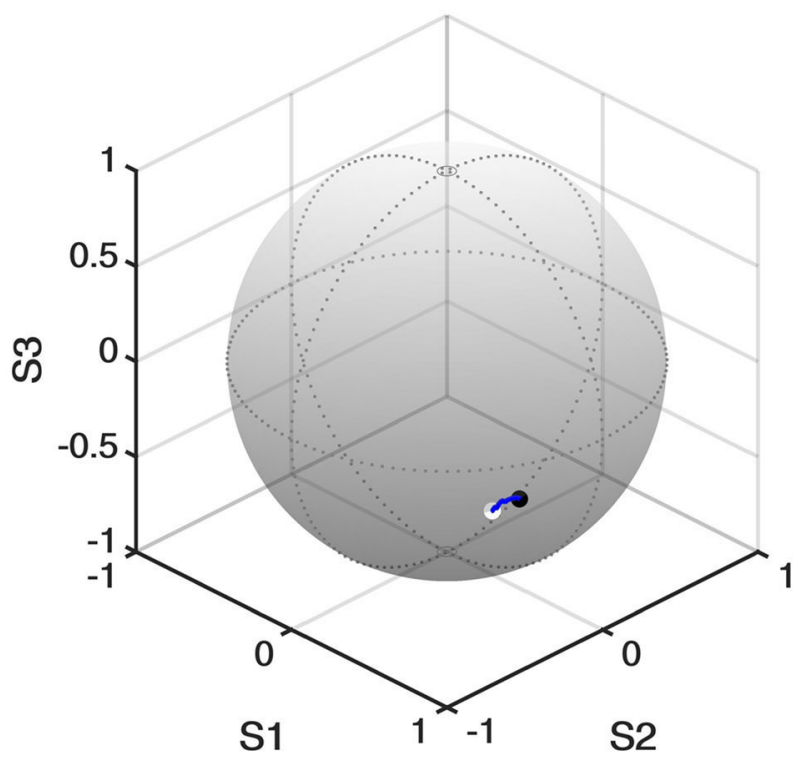

(b)

Figure 3. Polarization transformations on a Poincare sphere of the transmitted light in the presence of water molecules (polar) (a) and carbon dioxide (nonpolar) vapors (b). White dot: Initial state of polarization (SOP). Black dot: Final SOP. Blue line: SOP transformation path. S1, S2, S3: The three components of the Stokes vector, which defines the polarization of the light.

detected by monitoring the state of polarization (SOP) of the transmitted light through the silk strand. Hence, silk fibers are excellent for detecting polar-modifying agents such as water molecules, acids, and bases.

The experimental bench we used is illustrated in Figure 2. We launched a linearly polarized light into the silk fiber, which was kept under controlled temperature, humidity, and pressure conditions inside a gas chamber. We monitored the SOP of the corresponding transmitted light using a polarization analyzer.

When exposed to different polar molecules (water, acid, and ammonia), the SOP of the transmitted light changed instantaneously-as shown in Figure 3(a)-indicating a change in the fiber's birefringence. However, the presence of pure carbon dioxide gas, a nonpolar molecule, did not affect the silk fiber's birefringence: see Figure $3(\mathrm{~b}) .^{5}$

In summary, we have successfully demonstrated a proof-ofconcept optical fiber chemical sensing system using native spider silk. Our next step is to develop a compact, low-cost, and selective sensing system, which we will achieve only through a better understanding of how different classes of chemical compounds affect the molecular arrangement, and thus the optical properties of the silk threads. Unlike many novel materials today, silk can be produced at ambient conditions and can be functionalized for custom sensing applications by incorporating specific dyes in the bulk material. With recent advances in the industry-wide production of artificial spider silk, ${ }^{6}$ the possibility of having a new generation of cost-effective and biodegradable fibers with unique properties is gradually becoming a reality.

\section{Author Information}

Luc Thévenaz, Kenny Hey Tow, and Desmond Chow

École Polytechnique Fédérale de Lausanne

Lausanne, Switzerland

Luc Thévenaz is a professor leading a research group focused on photonics, specifically fiber optics and optical sensing. Research topics include fiber sensors, slow and fast light, nonlinear fiber optics, and laser spectroscopy in gases.

\section{Fritz Vollrath}

Department of Zoology

University of Oxford

Oxford, UK

\section{References}

1. M. Applegate, M. Brenckle, B. Marelli, H. Tao, D. Kaplan, and F. Omenetto, Silk: a different kind of fiber optics, Opt. Photon. News 25, pp. 28-35, 2014.

Continued on next page 
2. N. Huby, V. Vié, A. Renault, S. Beaufils, T. Lefèvre, F. Paquet-Mercier, M. Pézolet, and B. Bêche, Native spider silk as a biological optical fiber, Appl. Phys. Lett. 102, p. $123702,2013$.

3. F. Vollrath and D. P. Knight, Liquid crystalline spinning of spider silk, Nature 410, pp. 541-548, 2001.

4. D. M. Chow, K. Hey Tow, F. Vollrath, I. Dicaire, T. Gheysens, and L. Thévenaz, Shedding light on the optical properties of spider silk fiber, Proc. IEEE Photon. Conf. 2015, pp. 333-334, 2015.

5. K. Hey Tow, D. M. Chow, F. Vollrath, I. Dicaire, T. Gheysens, and L. Thévenaz, Spider silk: a novel optical fibre for biochemical sensing, Proc. SPIE 9634, p. 96347D, 2015. doi:10.1117/12.2192427

6. A. Rising and J. Johansson, Toward spinning artificial spider silk, Nat. Chem. Biol.

11, pp. 309-315, 2015. 